\title{
Effect of Step Depth and Angle on the Aerodynamics of a Sliding Morphing Skin
}

\author{
Fadi Mishriky ${ }^{*}$, Paul Walsh \\ Department of Aerospace Engineering, Ryerson University, Toronto, Canada \\ Email address: \\ Fadi.mishriky@ryerson.ca (F. Mishriky),paul.walsh@ryerson.ca (P. Walsh) \\ ${ }^{*}$ Corresponding author
}

To cite this article:

Fadi Mishriky, Paul Walsh. Effect of Step Depth and Angle on the Aerodynamics of a Sliding Morphing Skin. American Journal of Aerospace Engineering. Vol. 3, No. 3, 2016, pp. 24-30. doi: 10.11648/j.ajae.20160303.11

Received: September 19, 2016; Accepted: September 27, 2016; Published: October 18, 2016

\begin{abstract}
The past two decades have witnessed a growing interest among aerospace researchers and designers in aircraft morphing technology. A single aircraft with morphing wings can perform near optimum at different flight regimes by changing the geometry of its wings. With the advancements achieved in this field, a need for a reliable morphing skin is emerging. The demanding task of designing a morphing skin has to compromise between flexibility to ensure low actuation requirements, and high stiffness to carry all the aerodynamic loads. One of the viable designs that fulfills the mechanical requirements is the segmented sliding skin. In such a design, discrete panels overlap to cover the surface of the wing and slide against each other during the morphing motion. From the aerodynamic perspective, the sliding panels introduce backward-facing steps on airfoil surface. In the process of determining the optimum panels' thickness, this paper presents a comprehensive numerical study on the effect of the step depth and angle on the aerodynamics of an airfoil with a backward-facing step employed on its lower surface. Results showed a significant improvement in the lifting capabilities of the stepped airfoil, and this improvement is directly proportional to the step depth. On the other hand, the separated flow at the step edge induced a low pressure recirculation zone that created a suction force directly proportional to the effective area of the backward-facing step. This resulted in a drag coefficient value that is directly proportional to the step depth. The aerodynamic efficiency of the stepped airfoil was degraded in terms of the lift-to-drag ratio, however decreasing the step depth largely mitigated these adverse effects. Studying different step angles showed that the step can be tilted over a wide range of angles with a negligible effect on the aerodynamics of the stepped airfoil.
\end{abstract}

Keywords: Aerodynamics, Backward-Facing Step, Stepped Airfoil, Morphing Wing, Morphing Skin, Segmented Sliding Skin, Computational Fluid Dynamics

\section{Introduction}

Despite years of research and development in aviation, nearly all aircraft wings are designed according to the same model of a rigid structure equipped with few discrete control surfaces. Such design enables the aircraft to have optimum performance over a small range of flight conditions. The last couple of decades have witnessed a giant leap forward in the advancements of smart material and adaptive structures, which revived the attraction to a biomimetic concept that challenged this rigid model: morphing aircraft. In nature, birds are capable of morphing their wings and tails in a complex and fluid behaviour according to the desired flight regime [1], in the same manner, aircraft morphing technology enables a single air vehicle to undergo substantial geometric changes in-flight, with the purpose of increasing efficiency, versatility, and/or mission performance [2]. Sofla et al. [3] classified wing morphing to three main categories; airfoil profile adjustment, planform alternation (span change, chord length change and sweep angle change) and out-of-plane transformation (chord-wise bending, span-wise bending and wing twisting). Despite the attraction of this technology, aircraft morphing faces major challenges that hinder its technological readiness level. Reich and Sanders [4] listed a number of these challenges, but the most demanding of all is the developing of a morphing skin with all its diverse requirements. Kikuta [5] well outlines the requirements of a morphing skin; 
elastic/flexible in the direction of morphing to allow low force actuation, stiff enough to withstand aerodynamic and inertial loads, abrasion and chemical resistant, resistant to different weather conditions, high strain capability, high strain recovery rate and environmental longevity and fatigue resistance. With all the diverse requirements of a morphing skin, very few designs can be considered for the task. Thill et al. [6] presented a comprehensive review of several contemporary morphing skin technologies, and one of the viable designs that can fulfill most of the aforementioned requirements is the segmented sliding morphing skin. In such design, multiple discrete panels cover the outer shell of the wing and slide relative to each other during the morphing motion as shown in Fig. 1.

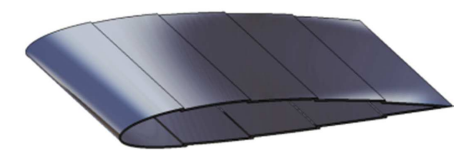

Figure 1. Segmented NACA 2412 airfoil with sliding morphing skin.

Ramrakhyani et al. [7] used a similar concept to develop a six-segmented morphing skin for a hydrofoil that is actuated with high frequency shape memory alloys (SMA). Air and water tunnel tests demonstrated the success of this sliding skin to accommodate all high frequency morphing motions. Instead of random or regular interval segmentation, Xijuan et al. [8] developed a systematic algorithm to segment the wing surface based on the change of airfoil curve before and after morphing. With most of the efforts in the morphing skin topic focused on the mechanical implementation and actuation, very few drew enough attention to the aerodynamic viability of such designs. The few discontinuities introduced at the panels' edges can interrupt the stability of the viscous boundary layer and alter the pressure distribution, leading to a dramatic change in the aerodynamic performance that can counteract all the potential gains of a morphing wing design. From the aerodynamic perspective, the interface between the sliding segments is regarded as backward-facing step incorporated along the chord-wise direction of the airfoil. In a previous study [9], the authors have studied the aerodynamic effect of employing a backward-facing step on the upper surface of a NACA 2412 airfoil. A correlation between the step location the aerodynamic properties was established to show that the step has decreased the lifting capability, increased the drag forces and lowered the critical angle of attack of the stepped airfoil. However, shifting the step from the leading edge towards the trailing edge can relatively mitigate these adverse effects. In the current study, a comprehensive numerical study will examine the aerodynamic effects of another geometric aspects of the backward-facing step, namely, the step depth and the step angle. High Reynolds number simulations will establish the correlation between the step angle and the step depth on the aerodynamic properties of a segmented morphing airfoil with a backward-facing step, this time, employed on the lower surface of the airfoil. First, the numerical model and boundary conditions will be presented, followed by a mesh independence study that utilized the Richardson's extrapolation method. Several configurations of the stepped airfoil will examine the effect of the step depth and angle on the lift coefficient $c l$, the drag coefficient $c d$ and the lift-to-drag ratio $L / D$. Finally, a conclusion will summarize the significant results of the study.

\section{Numerical Modeling and Accuracy Assessment}

\subsection{Numerical Modeling}

The flow around the airfoil was numerically modeled using the computational fluid dynamics (CFD) commercial code FLUENT V.15. This code uses the unstructured finite volume approach to solve the governing continuity, momentum, energy and turbulence equations on a discretized domain. An implicit density based solver was used with second order schemes to discretize the convection and diffusive fluxes of the transport equations. The least squares cell based method was used to reconstruct the gradients of the scalar quantities. For proper resolution of the viscous boundary layer and its transition from laminar to turbulent, the turbulence of the flow was modeled using the four equations Langtry-Menter transitional shear stress transport turbulence model (Transition-SST model). This turbulence model is also known as the $\gamma-R e_{\theta_{C}}-S S T$ model because it couples the $k-\omega$ SST model equations with two other equations, one for the intermittency $\gamma$ and the other for the momentum thickness Reynolds number $R e_{\theta_{C}}[10,11]$. Menter and Langtry $[11,12]$ tested this model on several aerodynamic applications such as a flat plate, a Zierke and Deutsch compressor, an NREL wind turbine, an Aerospatiale A and the McDonald Douglas 30P-30N airfoils. Results showed that the wall shear stresses were well resolved and perfectly matched the experimental data, an agreement that was not observed when a laminar model or fully turbulent models as the $k-\varepsilon$ or the $k-\omega$ models were used. For that reason, the transition SST model was chosen to numerically model the turbulence of the flow over the airfoil.

\subsection{Boundary Conditions}

The airfoil profile used in this analysis is a NACA (National Advisory Committee for Aeronautics) 2412 airfoil with a sharp trailing edge and a unity chord length. The lower surface of the airfoil follows the curvature of a NACA 2412 from the leading edge to the step location, afterwards, the Y-coordinates of the airfoil are scaled down to match the descent created by the step. Fig. 2 shows a schematic diagram of the stepped NACA 2412 airfoil overlaying the clean profile.

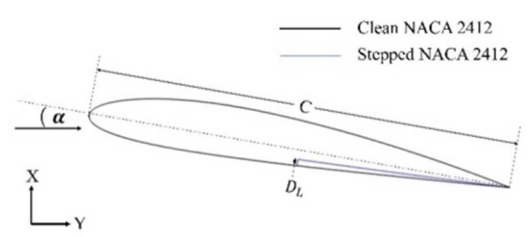

Figure 2. A schematic diagram of a clean NACA 2412 overlaying the stepped NACA 2412 airfoil. 
In all configurations, the step will be located at mid-chord length of the airfoil. For the effect of the step location, the reader can refer to [9]. The main stream fluid is directed at an angle of attack of $2.5^{\circ}$ and treated as an ideal gas at $300 \mathrm{~K}$, with its viscosity modeled using the Sutherland three coefficient method. The Reynolds number according to the chord length of the airfoil is $5.9 \times 10^{6}$. A Mach number lower than 0.1617 was used to match the experimental conditions documented by Abbot and Von Denhoff [13].

\subsection{Mesh Independence Study}

A C-mesh topology is used for the computational domain where the mesh lines follow the shape of the airfoil in a $\mathrm{C}$ shape. To ensure a proper density of the used mesh, a mesh independence study was performed using a family of five consecutively refined meshes. A refinement factor of $r=2$ was used, so the number of nodes is doubled in each direction, thus the number of cells is quadrupled from one mesh to the other. The flow was solved on each mesh and the lift coefficient $c_{l}$ was calculated to judge the independence of the solution from the mesh density. Fig. 3 shows the obtained values of the lift coefficient versus the number of cells in each mesh.

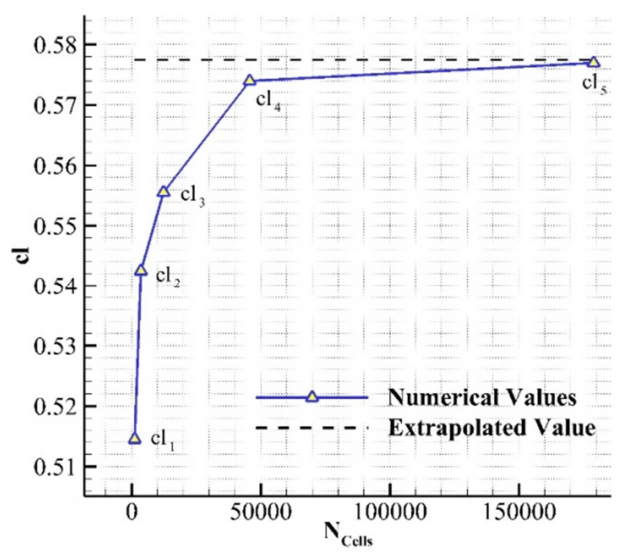

Figure 3. Convergence of the lift coefficient values as the density mesh increases.

Fig. 3 shows that as the number of cells increases, the error in the calculations approaches zero, and the lift coefficient value approaches an asymptotic value known as the continuum value $c_{l(h=0)}$. This value is a theoretical value that would be obtained if the spacing $h$ between the nodes approached zero $(h \rightarrow 0)$. The continuum value can be approximated using the Richardson's extrapolation method. This method uses the values of the lift coefficient obtained from the finest three meshes $\left(c_{l 3}, c_{l 4}\right.$ and $\left.c_{l 5}\right)$ to calculate the continuum value $c_{l(h=0)}$ as follows:

$$
c_{l(h=0)} \cong c_{l 5}+\frac{c_{l 5}-c_{l 4}}{r^{p}-1}
$$

where $r$ is the refinement ratio, and $p$ is the observed order of accuracy and is calculated as:

$$
p=\frac{\ln \left(\frac{c_{l 3}-c_{l 4}}{c_{l 4}-c_{l 5}}\right)}{\ln (r)}
$$

The continuum value calculated from equations (1) and (2) was found equal to 0.5775 which is less that $0.1 \%$ off the value obtained numerically from the finest mesh $\left(c_{l 5}\right)$. For that reason, the finest mesh which consists of about 180,000 cells will be used to carry out the numerical testing in the rest of this study. Any additional refinement of the mesh will negligibly improve the accuracy of the solution, but will consume a significant amount of computational time and resources to reach convergence.

\section{Results and Discussion}

\subsection{Effect of the Step Depth $D_{L}$}

In this subsection, the effect of the step depth on the aerodynamics of the stepped NACA 2412 airfoil will be examined. Eight different step depths are tested, ranging from $D_{L} / C=0.0075$ to $D_{L} / C=0.025$, with an increment of $0.0025 C$ from one configuration to the other. In all cases, the flow is directed with an angle of attack of $2.5^{\circ}$, and the step is located at the mid-chord length of the airfoil. Fig. 4 shows the two extreme tested configurations with step depths of $D_{L} / C=0.0075$ and 0.025 , respectively.

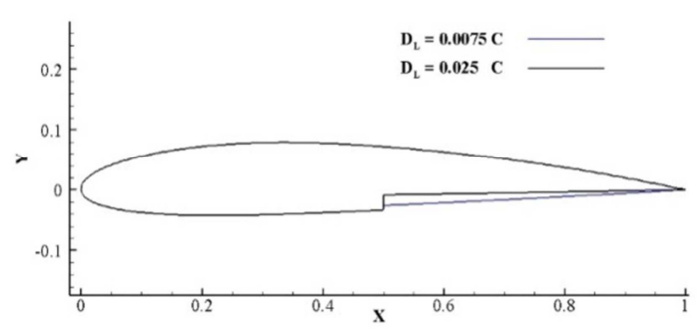

Figure 4. The two extreme step depths.

In each of the eight configurations, the lift coefficient $c_{l}$, the drag coefficient $c_{d}$, and the lift-to-drag ratio $L / D$ are calculated to establish the correlation between the step depth and each of the aforementioned aerodynamic properties.

As the step depth gradually increased from $D_{L} / C=$ 0.0075 to $D_{L} / C=0.025$, the lift coefficient increased $10 \%$ from 0.55 to 0.60 as shown in Fig. 5. In all cases, the lift coefficient value obtained from the stepped configuration is higher than 0.517 , which is the value obtained from the clean NACA 2412 at the same boundary conditions.

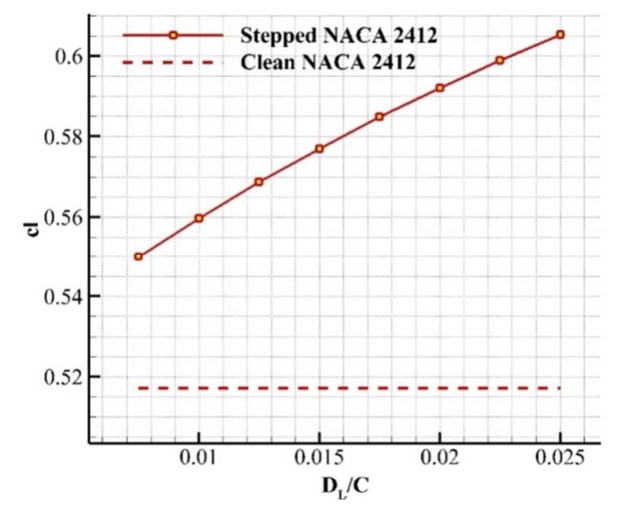

Figure 5. The lift coefficient value $\boldsymbol{c}_{\boldsymbol{l}}$ at different step depths. 
The stepped configurations have improved the lifting capability of the airfoil by $6 \%$ for a step depth of $D_{L} / C=$ 0.0075 , and increased with the step depth to reach $17 \%$ for a step depth of $D_{L} / C=0.025$. The improved lift is attributed to the decrease of the airfoil thickness caused by introducing a step on the lower surface of the airfoil. The backward-facing step caused the flow to separate at the step edge and created a low pressure recirculation zone at the step vicinity. The low pressure region assisted the flow to reattach again to the thinned lower surface of the airfoil. In a trial to fill in the extra space introduced by the step, the flow experienced a deceleration in the velocity governed by the conservation of mass, while the conservation of momentum dictated an increase in the static pressure values acting on the pressure side of the airfoil, thus enhancing its lifting forces.

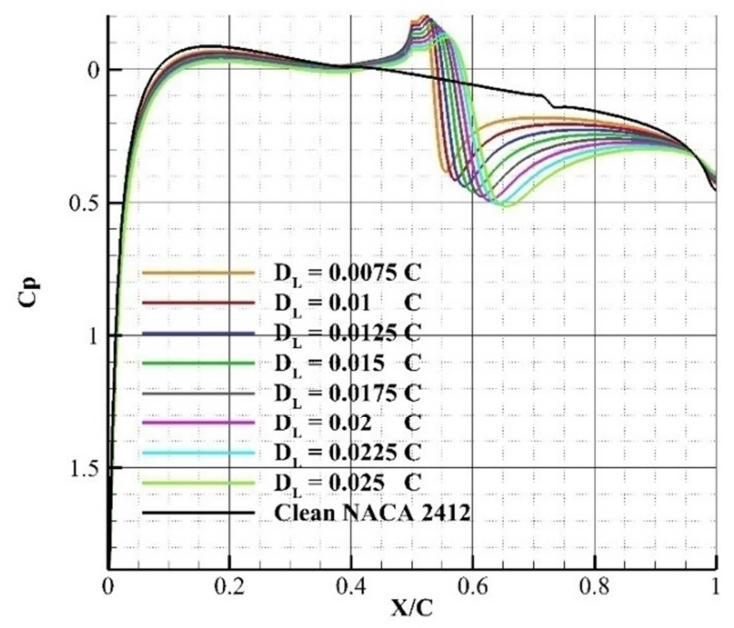

Figure 6. Pressure distribution over the lower surface of a clean NACA 2412 as well as in cases of stepped airfoil with different step depths.

Fig. 6 shows the pressure distribution over the lower surface of different configurations of the stepped airfoil, as well as on the clean airfoil. Comparing the pressure distribution of the clean airfoil with the stepped configurations shows that the stepped configuration is subjected to higher pressure values acting on its lower surface. This justifies the improved lift coefficient values experienced by the stepped configurations. As the step depth increases, the airfoil experiences higher pressure values that will cause the lift coefficient to increase with the increase of the step depth as shown in Fig. 5.

Despite the noticeable improvement in the lifting capabilities associated with introducing the step, a significant increase in the drag force is also experienced. Fig. 7 shows the variation of the drag coefficient value obtained with different step depths.

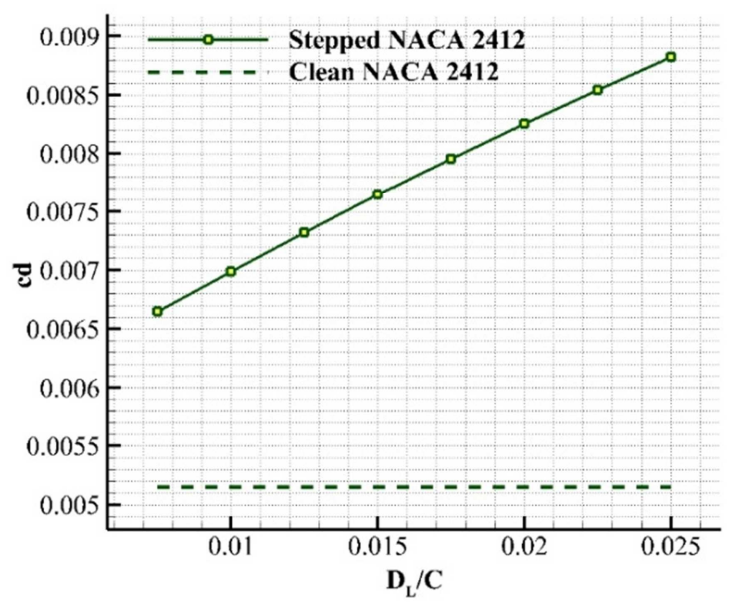

Figure 7. The drag coefficient value $\boldsymbol{c}_{\boldsymbol{d}}$ at different step depths.

A step with a depth of $D_{L} / C=0.0075$ resulted in a $29 \%$ increase in the drag forces when compared to the drag forces acting on the clean airfoil. This increase in the drag forces grows with the step depth to reach $71.36 \%$ in case of a step with a depth of $D_{L} / C=0.025$. To understand the correlation between the step depth and the drag forces, the drag coefficient value is decomposed using equation (3) to its two main components, namely, the pressure (form) drag coefficient and the viscous drag coefficient.

$$
c_{d}=c_{p}+c_{f}=\frac{1}{\rho v^{2} A} \int_{S}\left(p-p_{0}\right)(\hat{n} . \hat{\imath}) d A+\frac{1}{\rho v^{2} A} \int_{S} \tau_{w}(\hat{t} . \hat{\imath}) d A
$$

Where $c_{p}$ is the pressure drag coefficient, $c_{f}$ is the friction drag coefficient or viscous drag coefficient, $\rho$ is the fluid density, $v$ is the reference velocity, $A$ is the reference area, $p$ is the pressure at the surface $d A, p_{0}$ is the reference pressure, $\hat{n}$ is a unit vector normal to the surface $d A, \tau_{w}$ is the wall shear stresses at the surface $d A$ and $\hat{t}$ is a unit vector tangent to the surface $d A$. The pressure drag coefficient is the surface pressure integral component parallel to the direction of the main stream velocity, while the viscous drag component is calculated by integrating the wall shear stresses acting on the airfoil surface and decomposed to a component parallel to the main stream velocity. Results of this decomposition is shown in Fig. 8.

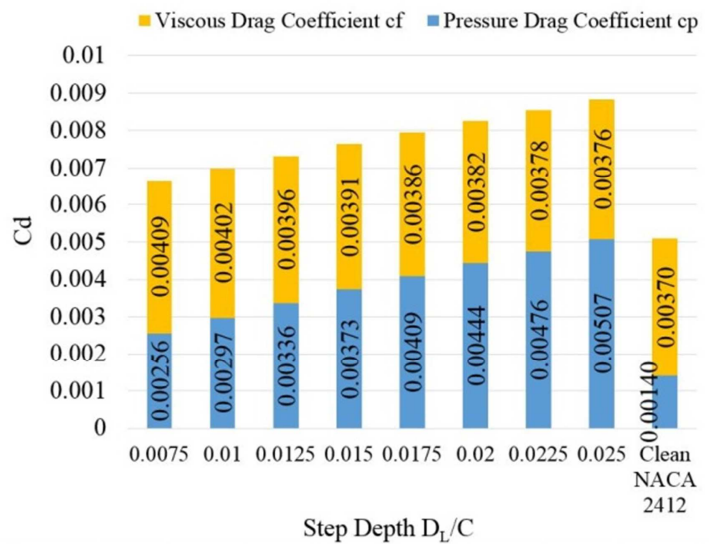

Figure 8. Decomposition of the Drag coefficient to pressure drag and viscous drag coefficients at different step depths. 
As the step depth increases, the viscous drag coefficient slightly decreases, but the pressure drag coefficient significantly increases from one step depth to the other. The viscous drag coefficient is directly proportional to the integral of the shear stresses acting on the walls of the airfoil, which in turn is function of the velocity gradient normal to the walls. For a laminar boundary layer, the velocity gradient has a negligible value when compared to the large gradient values experienced at a turbulent boundary layer. So as the transition of the boundary layer from laminar to turbulent is delayed, less viscous drag is experienced by the airfoil. Normally, the viscous boundary layer starts as a thin laminar boundary layer at the leading edge of the airfoil. As the flow approaches the mid chord length, the boundary layer experiences a number of instabilities that triggers the transition from laminar to turbulent. Such instabilities include wall roughness or obstruction, free stream turbulences, acoustic waves, pressure gradients, surface curvature and other. In case of the clean NACA 2412 , the natural transition occurred at about $73 \%$ of the chord length. The transition is shown in Fig. 9 as sudden increase in the value of the skin friction coefficient.

The transition in cases of the stepped configuration occurred at the reattachment point of the flow after the recirculation zone, which is before the natural transition point. For that reason, Fig. 8 shows less viscous drag value in case of the clean airfoil. In cases of the stepped airfoil, the viscous forces dropped slightly as the step depth increased since larger step depths generated more entropy at the recirculation zone which resulted in more energy dispersal, hence, lower turbulent kinetic energy after the reattached. This lower turbulent kinetic energy is translated to lower skin friction coefficient values shown in Fig. 9 for cases with larger step depths. For that reason, the viscous drag coefficient decreases as the step depth increases.

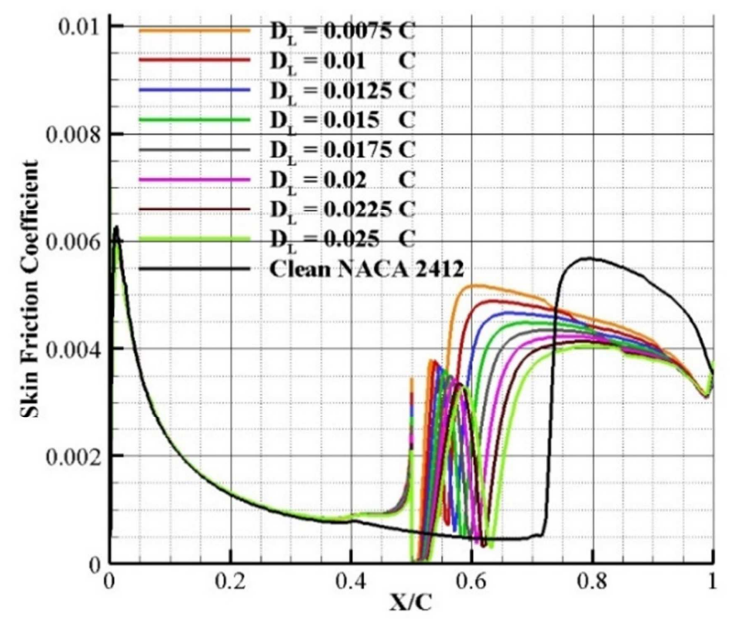

Figure 9. Skin friction coefficient distribution over the lower surface of a clean NACA 2412 as well as in cases of stepped airfoil with steps at different locations.

On the other hand, the pressure drag coefficient significantly increased with the step depth because the low pressure recirculation zone creates a suction force acting on the vertical wall of the backward-facing step. As the step depth increases, the effective area on which the low pressure region is acting increases. The result is higher suction forces and thus more pressure drag values. The increase in the pressure drag component exceeded the drop in the viscous drag component, leading to an overall increase in the value of the drag coefficient that increase with the step depth as shown in Fig. 7.

Another important aerodynamics efficiency metric is the lift-to-drag ratio $L / D$, which is the amount of lift generated by the airfoil divided by its aerodynamic drag. For any airfoil design, a higher lift-to-drag ratio is a major goal to achieve a better fuel consumption, higher glide ratio and improved climb. Fig. 10 shows the lift-to-drag $L / D$ ratio, the lift coefficient $c_{l}$ and the drag coefficient $c_{d}$ obtained at each step depth, as well as the value obtained from the clean airfoil.

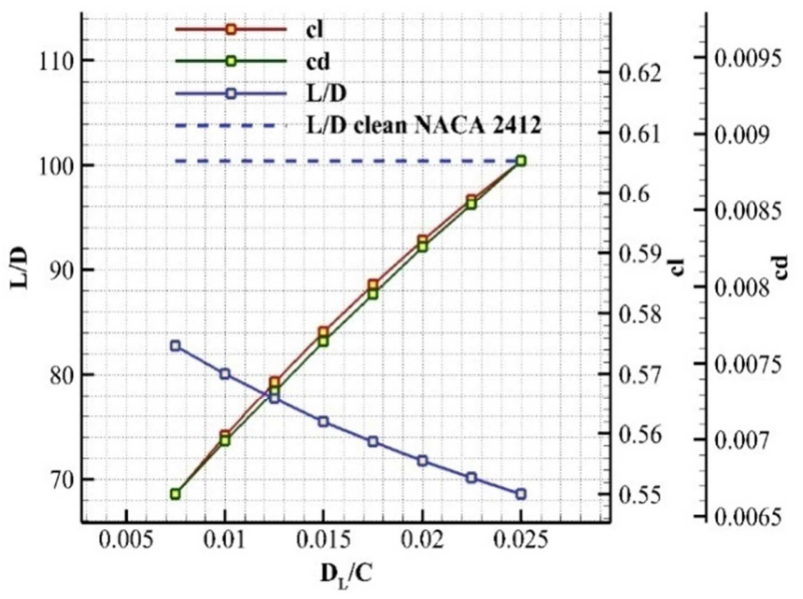

Figure 10. The lift-to-drag ratio $L / D$, the lift coefficient value $\boldsymbol{c}_{\boldsymbol{l}}$ and the drag coefficient value $\boldsymbol{c}_{\boldsymbol{d}}$ at different step depths.

As the step depth increases from $D_{L} / C=0.0075$ to $D_{L} /$ $C=0.025$, the lift-to-drag ratio drops, but in all cases, the obtained values are lower than 100.43 which is the value obtained by the clean airfoil at the same flight conditions. Despite the favorable effect of the increasing step depth on the lifting capabilities of the step, the increase in the drag coefficient values is more dominant than the increase in the lift values. It was observed that the variations in lift coefficient value from one step depth to the other is in the order of magnitude of 10-3, while the changes in the drag coefficient are in the order of 10-4. Therefore, when calculating the lift-to-drag ratio, the correlation between the drag coefficient and the lift-to-drag ratio will be nearly 10 times larger than the correlation between the lift coefficient and the lift-to-drag ratio. For that reason, the lift-to-drag ratio follows an inverse relation with the drag coefficient, and thus with the step depth. This means that as the thickness of sliding skin panels decreases, higher lift-to-drag ratio can be achieved, but in call cases, the aerodynamic efficiency in terms of the lift-to-drag ratio is lower than the clean airfoil.

\subsection{Effect of the step angle $\beta$}

This subsection focuses on the influence of the backward-facing step angle $\beta$ on the aerodynamic properties 
of the stepped airfoils. Changing the step angle may affect the shape of the recirculation zone and its intensity, thus affect the aerodynamic performance of the stepped airfoil. Five different step angles are tested ranging from $\beta=45^{\circ}$ to $\beta=-45^{\circ}$ as shown in Fig. 11, where a positive angle is measured from lower edge of the step and in the counter-clockwise direction.

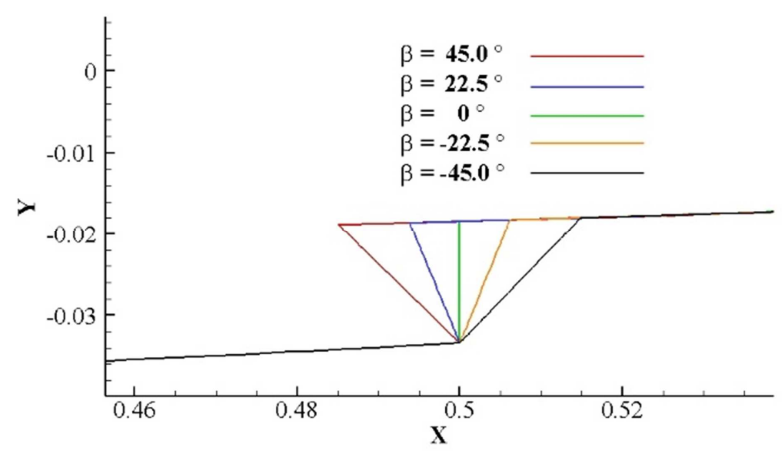

Figure 11. Stepped configurations of the NACA 2412 with different step angles.

The flow was numerically solved in each of the five cases, and values of the lift coefficient, drag coefficient and lift-to-drag ratio are calculated. Table 1 shows the values of the aforementioned properties at different step angles.

Table 1. Values of $c l, c d$ and $L / D$ at different step.

\begin{tabular}{lccc}
\hline \multicolumn{1}{c}{$\boldsymbol{\beta}$} & $\boldsymbol{c}_{\boldsymbol{l}}$ & $\boldsymbol{c}_{\boldsymbol{d}}$ & $\boldsymbol{c}_{\boldsymbol{l}} / \boldsymbol{c}_{\boldsymbol{d}}$ \\
\hline $45.0^{\circ}$ & 0.57703 & 0.0076517 & 75.41296 \\
$22.5^{\circ}$ & 0.57698 & 0.0076474 & 75.44916 \\
$0^{\circ}$ & 0.57697 & 0.0076459 & 75.46084 \\
$-22.5^{\circ}$ & 0.57699 & 0.0076473 & 75.45039 \\
$-45.0^{\circ}$ & 0.57706 & 0.0076571 & 75.36398 \\
\hline
\end{tabular}

There is a negligible difference in the lift coefficient, drag coefficient and lift-to-drag ratio values from one step angle to the other. This can be explained by comparing the skin friction coefficient distribution of each case as shown in Fig. 12

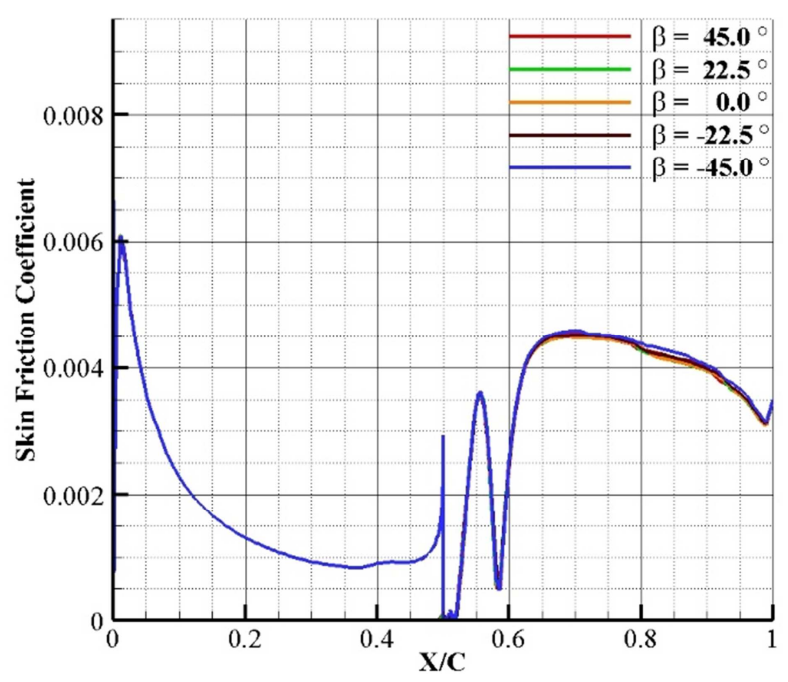

Figure 12. Skin friction coefficient of the five configurations with different step angles.
The five configurations obtained the same skin friction coefficient distribution regardless of the step angle. In all cases, the recirculation zone started at $X / C=0.52$ and ended at $X / C=0.585$ even at a step angle of $\beta=45^{\circ}$ which creates the largest vicinity at the step corner. This is attributed to the generation of a cascade of small scale, low energy eddies that fill in any additional space created by changing the step angle. These eddies are known as the Moffatt eddies, and theoretically there should be infinite number of them trapped at the step corner as their length scale decreases to zero. As the computational domain is discretized with a denser mesh, the number of modelled Moffatt eddies increases. Fig. 13 shows the streamlines of the eddies induced by the main recirculation zone at different step angles.
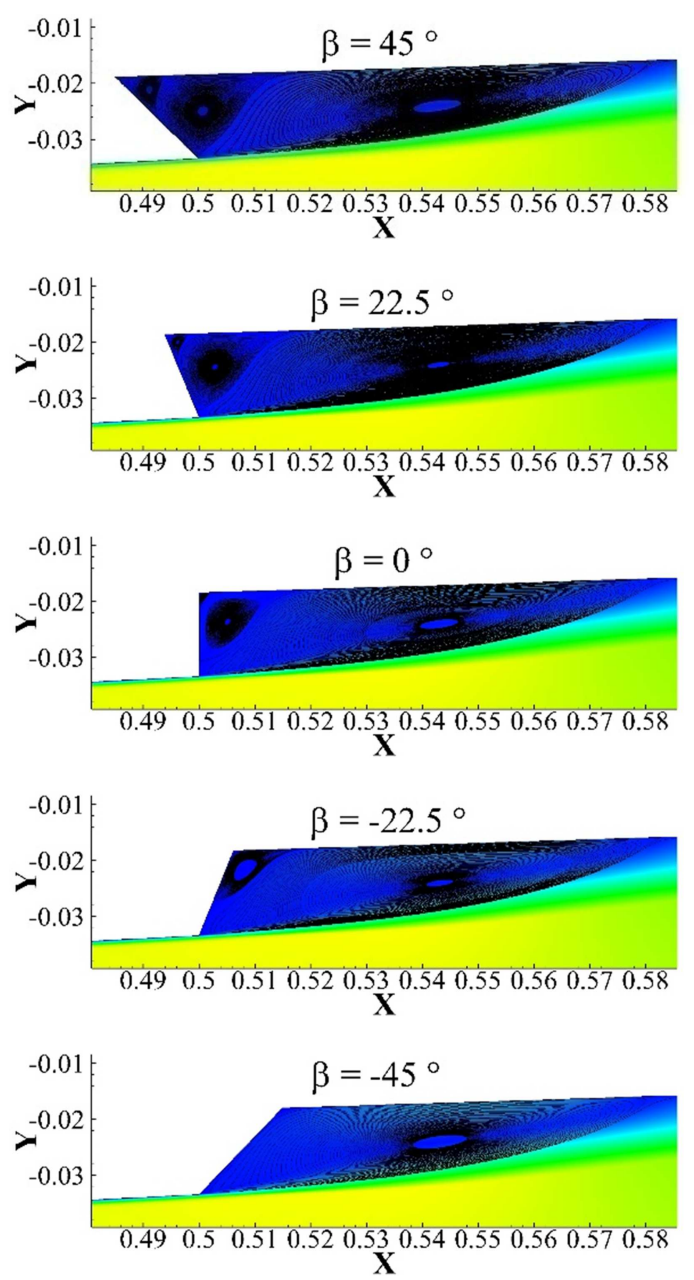

Figure 13. Streamlines of the recirculation zone and the Moffatt eddies at different step angles.

Fig. 13 shows that in the five cases, the main recirculation zone has the same structure and size regardless of the step angle. As the step angle increased from $\beta=-45^{\circ}$ to $\beta=$ $45^{\circ}$, an additional space is created at the step corner. However, the extra space created by tilting the step edge with different angles is filled by the Moffatt eddies. Fig. 13 shows that in case of a step angle of $\beta=45^{\circ}$, four levels of eddies are modeled, and the number of eddies decreases as the step angle decreases. Among the different levels of eddies, only the main recirculation zone has an influence on the aerodynamics of the 
airfoil, while the Moffatt eddies are low energy secondary flows with negligible effect on the aerodynamic performance. For that reason, lift coefficient, drag coefficient and lift-to-drag ratio are not influenced by the variation of the step angle $\beta$.

\section{Conclusion}

In the process of designing a viable sliding skin for a morphing wing, the effect of the step depth and angle on the aerodynamics of a stepped NACA 2412 airfoil has been numerically analyzed. The lift coefficient, drag coefficient and the lift-to-drag is calculated for eight different configurations with different depths. Results showed employing a step on the lower surface of an airfoil has improved its lifting capabilities by at least $6 \%$ for a step depth of $D_{L} / C=0.0075$, and increased with the step depth to reach $17 \%$ for a step depth of $D_{L} / C=0.025$. On the other hand, the separation of the flow at the step edge triggered the transition of the boundary layer from laminar to turbulent and created a low pressure recirculation zone which significantly increased the drag coefficient value. The increase of the drag forces negated the favorable effect of the step on the lifting force, and resulted in lower aerodynamic efficiency in terms of the lift-to-drag ratio. The hindered performance of the stepped configurations can be relatively improved by decreasing the step depth. Studying different step angles showed that the angle has a negligible effect on the aerodynamic properties of the airfoil because any additional space created at the step corner will be filled with low energy eddies known as the Moffatt eddies, which have insignificant influence on the flow field. Thus the edge of the sliding panels can be tilted at any angle, but the minimum allowable thickness of the panels should be used, as any increase in the thickness will degrade the aerodynamic performance of the morphing wing.

\section{Nomenclature}

$p$ : Observed Order of accuracy

$C_{p}$ : Pressure Coefficient

$X_{L}$ : Step location on the lower surface of the airfoil

$c_{d}$ : Drag coefficient

$c_{f}$ : Viscous (friction) drag coefficient

$c_{l}:$ Lift coefficient

$c_{p}$ : Pressure drag coefficient

$\hat{n}$ : unit vector normal to the surface

$p_{0}$ : Reference pressure

$\hat{t}$ : unit vector tangent to the surface

$\tau_{w}:$ Wall shear stresses

$\mathcal{F}$ : Aerodynamic property used in the Richardson's Extrapolation method

\author{
$A$ : Reference Area \\ $C$ : Chord length of the airfoil \\ $k$ : Specific turbulent kinetic energy \\ $r$ : Mesh refinement factor \\ $v$ : Reference velocity of the flow \\ $\varepsilon$ : Turbulent kinetic energy dissipation rate \\ $\rho$ : Density of the fluid \\ $\omega$ : Specific dissipation rate
}

\section{References}

[1] A. Wickenheiser, and E. Garcia. Aerodynamic modeling of morphing wings using an extended lifting-line analysis. Journal of Aircraft. 2007 Jan;44(1):10-6.

[2] A. Wickenheiser, E. Garcia, and M. Waszak. Evaluation of bio-inspired morphing concepts with regard to aircraft dynamics and performance. In Smart Structures and Materials 2004 Jul 26 (pp. 202-211).

[3] A. Y. Sofla, S. A. Meguid, K. T. Tan, and W. K. Yeo. Shape morphing of aircraft wing: status and challenges. Materials \& Design. 2010 Mar 31;31(3):1284-92.

[4] G. Reich, and B. Sanders. Introduction to morphing aircraft research. J Aircraft 2007;44:1059.

[5] M. T. Kikuta. Mechanical properties of candidate materials for morphing wings, 2003, p 123, Department of Mechanical Engineering, Virginia Polytechnic Institute and State University.

[6] C. Thill, J. Etches, I. Bond, K. Potter, and P. Weaver. Morphing skins. The Aeronautical Journal 2008, 112(1129), 117-139.

[7] D. S. Ramrakhyani, G. A. Lesieutre, M. I. Frecker, and S. Bharti. Aircraft structural morphing using tendon-actuated compliant cellular trusses. Journal of aircraft. 2005 Nov;42(6):1614-20.

[8] G. Xijuan, Z. Qiang, and F. Xi. Design Segmented Stiff Skin for a Morphing Wing. Journal of Aircraft. 2015 Dec 22:1-9.

[9] F. Mishriky, and P. Walsh. Effect of the Backward-Facing Step Location on the Aerodynamics of a Morphing Wing. Aerospace $2016,3,25$.

[10] R. B. Langtry, and F. R. Menter. Correlation-based transition modeling for unstructured parallelized computational fluid dynamics codes. AIAA journal. 2009 Dec; 47(12):2894-906.

[11] F. R. Menter, R. Langtry, and S. Völker. Transition modelling for general purpose CFD codes. Flow, turbulence and combustion. 2006 Nov 1;77(1-4):277-303.

[12] R. B. Langtry, and F. Menter. Transition modeling for general CFD applications in aeronautics. AIAA paper. 2005 Jan 10;522(2005):14.

[13] I. H. Abbott, and A. E. Von Doenhoff. Theory of wing sections, including a summary of airfoil data. Courier Corporation, 1959. 